\title{
Birth, death, and revival of spontaneous emission in a three-atom system
}

\author{
Simon Mährlein, ${ }^{1,2}$ Lukas Götzendörfer ${ }^{1,3}$ Kevin Günthner, ${ }^{4,1}$ Jörg Evers, ${ }^{5}$ and Joachim von Zanthier ${ }^{1,2,3, *}$ \\ ${ }^{1}$ Institut für Optik, Information und Photonik, Staudtstr. 1, Universität Erlangen-Nürnberg, 91058 Erlangen, Germany \\ ${ }^{2}$ Erlangen Graduate School in Advanced Optical Technologies (SAOT), Paul-Gordan-Str. 6, \\ Universität Erlangen-Nürnberg, 91052 Erlangen, Germany \\ ${ }^{3}$ International Max Planck Research School for the Science of Light (IMPRS-PL), Staudtstr. 2, 91058 Erlangen, Germany \\ ${ }^{4}$ Quantum Information Processing Group (QIV), Max Planck Institute for the Science of Light (MPL), Staudtstr. 2, 91058 Erlangen, Germany \\ ${ }^{5}$ Max Planck Institute for Nuclear Physics, Saupfercheckweg 1, 69117 Heidelberg, Germany
}

(Received 13 September 2019; accepted 24 January 2020; published 9 March 2020)

\begin{abstract}
Three identical two-level atoms in free space prepared in particular entangled single-photon excited states display a "birth," "death," and a nonperiodic "revival" of spontaneous emission in selected directions. Instead of recording the spontaneously emitted photon with a maximum probability at $t=0$ as for a single atom, a "birth" manifests itself in an initially zero photon detection probability, increasing thereafter in particular directions. Alternatively, the photon detection probability decreases in particular directions from an initially maximal value to completely disappear ("death") and to reappear again ("revival"). We show how these phenomena can be induced in the fully excited system, by projecting the atomic ensemble into the required entangled single-photon excited state via detection of the first two spontaneously emitted photons. To observe death and revival of spontaneous emission it is necessary to provide both spatial and temporal interference for which a minimum of three atoms is required. Hereby, the third atom, located at a large distance with respect to the other two atoms, can be used to tune the time and direction of the death of the photon. From this manipulation of spontaneous decay at a distance, we anticipate multiple applications, in fundamental science as well as in quantum technologies.
\end{abstract}

DOI: 10.1103/PhysRevResearch.2.013278

Collapse and revival occur for many phenomena in quantum physics appearing in different aspects and forms [1-7]. Typically, an observable of a system falls off in time to vanish at a given moment only to reappear again, in some cases even to its original value, sometimes in a periodic manner. A prominent example from atomic physics is a two-level atom interacting with a single mode of a cavity, as described by the Jaynes-Cummings model [1]. Here, in the absence of losses, the atomic inversion [2] as well as the atomic dipole moment [3] display periodic collapses and revivals, what has been observed also experimentally [4,5]. Another well-known example are spin echos [6], where the spins of an inhomogenously broadened atomic ensemble precess at a different pace in an external magnetic field, producing after some time a zero total magnetization; yet, a suitable inversion pulse can effectively reverse the dephasing process leading after some time to a revival of the initial magnetization.

A common feature of collapse and revival is that it typically occurs in closed systems, e.g., subject to particular boundary conditions. Here, lossy channels, as the infinite amount of vacuum modes in spontaneous decay, are typically not involved. Indeed, losses tend to prohibit rephasing and revivals in a

\footnotetext{
*joachim.vonzanthier@physik.uni-erlangen.de

Published by the American Physical Society under the terms of the Creative Commons Attribution 4.0 International license. Further distribution of this work must maintain attribution to the author(s) and the published article's title, journal citation, and DOI.
}

finite time, rather inducing a complete collapse of the wave function of the observables in question [8]. Moreover, while dephasing-based collapses and revivals occur commonly in a finite time, the infinite number of loss channels in open systems leads to an exponential decay and therefore requires an infinite amount of time for a complete collapse [9].

An interesting link between collapse and revival due to deand rephasing versus collapses induced by lossy channels has recently been discussed in the context of quantum entanglement [10]. Here, the dynamics of two entangled atoms was studied under the influence of spontaneous decay. It was found that even though the spontaneous emission process of the individual atoms requires an infinite time, the entanglement itself between the atoms can vanish within a finite time interval leading to a so-called "sudden death of entanglement" [10]. Later it was shown that after the sudden death there exist even conditions for which the entanglement reappears, despite the intrinsically incoherent nature of spontaneous emission $[9,11-13]$.

Here, we go one step further, and ask the question whether spontaneous emission itself can collapse in a finite time interval and subsequently exhibit revivals. Since the atomic excitation is coupled to an infinite number of vacuum modes, rephasing is not to be expected. Yet, in this paper, we show that three identical two-level atoms in free space, sharing a single excitation coherently among all particles, can indeed display a birth or, alternatively, a death and a nonperiodic revival of spontaneous emission. Starting from an initially fully excited nonentangled three-atom system, these counterintuitive phenomena can be induced by projecting the system 


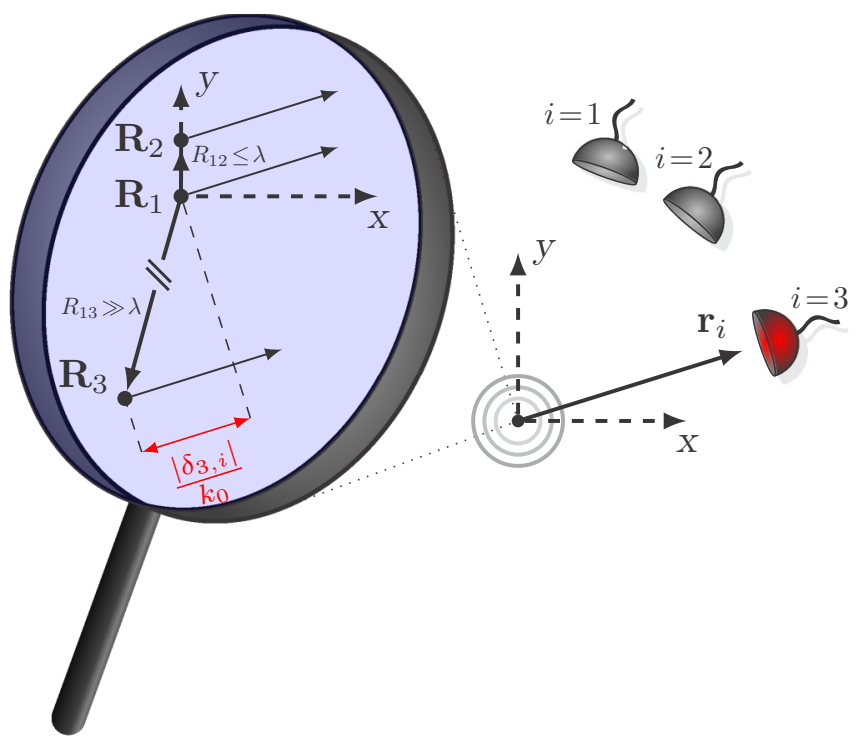

FIG. 1. Geometry of the investigated setup. Three identical twolevel atoms are located in free space in the $x-y$ plane at $\mathbf{R}_{1}, \mathbf{R}_{2}$, and $\mathbf{R}_{3}$ with dipole moments oriented perpendicular to that plane. Atoms 1 and 2 are placed close to each other so that they are subject to the dipole-dipole interaction, while atom 3 is located far from the other two atoms. Three detectors at $\mathbf{r}_{i}, i=1,2,3$, record the spontaneously emitted photons in the far field. By measuring two photons coincidentally at $\mathbf{r}_{1}$ and $\mathbf{r}_{2}$ from the fully excited system, the atomic ensemble is transferred into the entangled state $\hat{\rho}_{\text {sub }}$ via projection. The signal of the third detector (red) corresponds to the probability to record the last photon at $t_{3}$ and $\mathbf{r}_{3}$, spontaneously scattered by the entangled atomic ensemble.

into the required entangled state via the detection of two spontaneously emitted photons. A "birth" corresponds to a zero probability to detect a photon spontaneously emitted from the atomic ensemble at a time $t=0$, increasing thereafter to a maximal value at $t>0$. Alternatively, the detection probability may decrease from an initially maximal value to completely disappear at $t>0$ ("death"), only to reappear again ("revival"). As it turns out, the latter behavior occurs for a minimum of three atoms only, when two atoms are separated by a distance small compared to the transition wavelength $\lambda$, and a third atom is located far away from the other two particles. Only in such a configuration both spatial interference (via the remote atom) and temporal interference (via the two interacting collective emitting atoms) occur at the same time. In contrast, death and revival can not be observed with merely two atoms.

To investigate the phenomenon, we study the setup displayed in Fig. 1. Here, three identical two-level atoms are located in the $x-y$ plane at three different positions and three detectors placed in the far field of the atoms record the photons spontaneously scattered by the atomic ensemble. All three atoms are fully excited initially. We do not consider external driving fields, but the option that the atoms are transferred into the required entangled state via projective measurements $[14,15]$, i.e., by recording two photons coincidentally at $t=0$ at positions $\mathbf{r}_{1}$ and $\mathbf{r}_{2}$, emitted from the fully excited system. After this measurement, the atomic ensemble is left with a single excitation, shared coherently among all three atoms. The resulting state is described by the density matrix $[16,17]$

$$
\hat{\rho}_{\text {sub }}=A \hat{D}_{2} \hat{D}_{1}|e, e, e\rangle\langle e, e, e| \hat{D}_{1}^{\dagger} \hat{D}_{2}^{\dagger},
$$

where the constant $A$ ensures normalization and the detection operator $\hat{D}_{m}$, removing a single excitation from the ensemble, is defined as $[16,17]$

$$
\hat{D}_{m}=\sum_{n=1}^{3} \mathrm{e}^{\mathrm{i} \delta_{n, m}} \hat{\sigma}_{n}^{-} .
$$

Here, $\hat{\sigma}_{n}^{-}$is the atomic lowering operator acting on the $n$-th atom, $k_{0}=2 \pi / \lambda=\omega_{0} / c$ is the wave number of the spontaneously emitted photons, and $\delta_{n, m}=-k_{0} \frac{\mathbf{R}_{n} \cdot \mathbf{r}_{m}}{\left|\mathbf{r}_{m}\right|}$ defines the phase accumulated by a photon emitted from an atom at $\mathbf{R}_{n}$ and recorded by a detector at $\mathbf{r}_{m}$, relative to a photon emitted at the origin (see Fig. 1). We further define $\mathbf{R}_{12}=\mathbf{R}_{1}-\mathbf{R}_{2}$ and suppose that the dipole moments of the atoms are all oriented perpendicular to the $x-y$ plane.

We next assume that two atoms of the ensemble are placed close to each other, i.e., $R_{12}=\left|\mathbf{R}_{12}\right| \leqslant \lambda$, so that they are subject to dipole-dipole interaction, while the third atom is located far from the other two atoms such that $R_{3 i}=$ $\left|\mathbf{R}_{3}-\mathbf{R}_{i}\right| \gg \lambda, i=1,2$. In this way, the third atom can be treated as independent.

We note that three nearby atoms have been studied previously [18]; these, however, do not exhibit the new effects we report here for our system of two nearby and one remote atom. For this configuration, we study the probability to record the last spontaneously emitted photon at $\mathbf{r}_{3}$, i.e., the first-order photon correlation function

$$
G_{\hat{\rho}_{\text {sub }}}^{(1)}\left(t_{3}, \mathbf{r}_{3}\right) \propto\left\langle\hat{E}_{3}^{(-)} \hat{E}_{3}^{(+)}\right\rangle_{\hat{\rho}_{\text {sub }}},
$$

where $\hat{E}_{m}^{(+)}\left(\hat{E}_{m}^{(-)}=\left[\hat{E}_{m}^{(+)}\right]^{\dagger}\right)$ denotes the positive (negative) frequency part of the electric field operator at $\mathbf{r}_{m}$, and $\langle\hat{A}\rangle_{\hat{\rho}_{\text {sub }}}$ is the expectation value of the operator $\hat{A}$ for the atomic ensemble being in the state $\hat{\rho}_{\text {sub }}$.

Since $\hat{E}_{m}^{(+)}\left(\hat{E}_{m}^{(-)}\right)$can be linked to the atomic lowering (rising) operators via $\hat{E}_{m}^{(+)} \propto \sum_{n=1}^{3} \mathrm{e}^{\mathrm{i} \delta_{n, m}} \hat{\sigma}_{n}^{-}$[19], we conclude from Eqs. (1)-(3) that the detection probability of the two-photon subtracted state can be equally derived from the third-order photon correlation function of the fully excited system $[16,17]$

$$
\begin{aligned}
& G_{\hat{\rho}_{\mathrm{exc}}}^{(3)}\left(0,0, t_{3} ; \mathbf{r}_{1}, \mathbf{r}_{2}, \mathbf{r}_{3}\right) \\
& \quad \propto\left\langle\hat{E}_{1}^{(-)} \hat{E}_{2}^{(-)} \hat{E}_{3}^{(-)} \hat{E}_{3}^{(+)} \hat{E}_{2}^{(+)} \hat{E}_{1}^{(+)}\right\rangle_{|e, e, e\rangle\langle e, e, e|} \\
& \quad \propto\left\langle\hat{E}_{3}^{(-)} \hat{E}_{3}^{(+)}\right\rangle_{\hat{\rho}_{\text {sub }}} .
\end{aligned}
$$

Note that the coupling between the first two atoms can be conveniently described within the Dicke basis, i.e., using the double excited, symmetric, antisymmetric, and ground states $|E\rangle=|e, e\rangle,|S\rangle=\frac{1}{\sqrt{2}}(|e, g\rangle+|g, e\rangle)$, $|A\rangle=\frac{1}{\sqrt{2}}(|e, g\rangle-|g, e\rangle)$, and $|G\rangle=|g, g\rangle$, respectively [19]. Hereby, the decay rate towards and from the symmetric state $|S\rangle$ [antisymmetric state $|A\rangle]$ is modified from $2 \gamma$ to $2(\gamma+$ $\Delta \gamma)$ [from $2 \gamma$ to $2(\gamma-\Delta \gamma)$ ], depending on the interatomic distance $R_{12}$, whereas the energy level is shifted by $+\hbar \Delta \Omega$ $(-\hbar \Delta \Omega)$ with respect to the unaffected atomic transition frequency $\omega_{0}[20,21]$. Both, the coupling constants and the 
corresponding master equation governing the time evolution of the system are given in Appendix A.

Before we investigate the photon detection probabilities $G_{\hat{\rho}_{\text {sub }}}^{(1)}\left(t_{3}, \mathbf{r}_{3}\right)$ for the three-atom system, let us start by studying a system consisting of two atoms only, closely separated by a distance $R_{12} \leqslant \lambda$. This will help us to appreciate the impact of the third independent atom on the probability to detect the last photon spontaneously emitted by the three-atom ensemble along particular directions, investigated thereafter.

For the two-atom system, the photon detection probability for the last photon spontaneously emitted by two atoms being in a one-photon subtracted state can again be conveniently calculated from the second-order photon correlation function of the fully excited system $[16,17]$. One finds $[22,23]$

$$
\begin{aligned}
G_{\text {2atoms }}^{(1)}\left(t_{2}, \mathbf{r}_{2}\right) \propto & \mathrm{e}^{-2 \gamma t_{2}}\left(\mathrm{e}^{-2 \Delta \gamma t_{2}} \cos ^{2}\left(\delta_{2,1} / 2\right) \cos ^{2}\left(\delta_{2,2} / 2\right)\right. \\
& +\mathrm{e}^{2 \Delta \gamma t_{2}} \sin ^{2}\left(\delta_{2,1} / 2\right) \sin ^{2}\left(\delta_{2,2} / 2\right) \\
& \left.+\sin \left(\delta_{2,1}\right) \sin \left(\delta_{2,2}\right) \frac{\cos \left(2 \Delta \Omega t_{2}\right)}{2}\right)
\end{aligned}
$$

where the sign of the last term in Eq. (5) can be positive or negative depending on the values of $\delta_{2,1}$ and $\delta_{2,2}$. In particular, for $t_{2}=0$ and $\delta_{2,1}=\delta_{2,2}+\pi(2 m+1), m \in \mathbb{Z}$, this leads to a complete destructive interference of all terms and vanishing of Eq. (5). Under these conditions, a birth of the photon detection probability is obtained, i.e., a signal starting from zero at $t_{2}=0$ and increasing for $t_{2}>0$ up to a maximal value, decreasing thereafter. However, from Eq. (5), it is not possible to derive a death of the photon detection probability, i.e., $G_{2 \text { atoms }}^{(1)}\left(t_{2}, \mathbf{r}_{2}\right)=0$ for $t_{2}>0$ as demonstrated in Appendix B. As it turns out, to achieve this an additional third atom is required.

We thus now turn to the three-atom case (see Fig. 1). The most general two-photon subtracted state of a pure state as in Eq. (1), can be written in the form

$$
\hat{\rho}_{\text {sub }}(0)=\left(c_{G e}|G, e\rangle+c_{S g}|S, g\rangle+c_{A g}|A, g\rangle\right) \cdot \text { H.c., }
$$

where we used the states $|\{G, S, A, E\}\rangle$ of the Dicke basis for the first two atoms and the bare basis $|\{g, e\}\rangle$ for the third atom. In Eq. (6), the coefficients $c_{G e}, c_{S g}$, and $c_{A g}$ are given by

$$
\begin{aligned}
& c_{G e}=\sqrt{A}\left(\mathrm{e}^{\mathrm{i} \delta_{2,1}}+\mathrm{e}^{\mathrm{i} \delta_{2,2}}\right), \\
& c_{S g}=\sqrt{\frac{A}{2}}\left(\mathrm{e}^{\mathrm{i}\left(\delta_{3,1}+\delta_{2,2}\right)}+\mathrm{e}^{\mathrm{i}\left(\delta_{2,1}+\delta_{3,2}\right)}+\mathrm{e}^{\mathrm{i} \delta_{3,1}}+\mathrm{e}^{\mathrm{i} \delta_{3,2}}\right), \\
& c_{A g}=\sqrt{\frac{A}{2}}\left(\mathrm{e}^{\mathrm{i}\left(\delta_{3,1}+\delta_{2,2}\right)}+\mathrm{e}^{\mathrm{i}\left(\delta_{2,1}+\delta_{3,2}\right)}-\mathrm{e}^{\mathrm{i} \delta_{3,1}}-\mathrm{e}^{\mathrm{i} \delta_{3,2}}\right)
\end{aligned}
$$

and depend, apart from the atomic geometry assumed to be fixed, only on the position of the first two detectors.

Since the time evolution of a single atom and two dipoledipole interacting atoms is well known [19], we obtain as a solution for the combined system

$$
\begin{aligned}
\hat{\rho}_{\mathrm{sub}}\left(t_{3}\right)= & \left(\mathrm{e}^{-\gamma t_{3}} c_{G e}|G, e\rangle+\mathrm{e}^{-(\gamma+\Delta \gamma+\mathrm{i} \Delta \Omega) t_{3}} c_{S g}|S, g\rangle\right. \\
& \left.+\mathrm{e}^{-(\gamma-\Delta \gamma-\mathrm{i} \Delta \Omega) t_{3}} c_{A g}|A, g\rangle\right) \cdot \text { H.c. } \\
& +c_{G g}\left(t_{3}\right)|G, g\rangle\langle G, g| .
\end{aligned}
$$

From Eq. (8), we see that the independent third atom decays with the unmodified decay rate $\gamma$ [first term in Eq. (8)], whereas for the first and second atoms, modified decay rates $\gamma \pm \Delta \gamma$ and energy shifts $\Delta \Omega$ appear in the exponents in front of the states $|S\rangle$ and $|A\rangle$ [second and third terms in Eq. (8)]. We also note that the explicit form of $c_{G g}\left(t_{3}\right)$ (fourth term in Eq. (8)) is not relevant for $G_{\hat{\rho}_{\text {sub }}}^{(1)}\left(t_{3}, \mathbf{r}_{3}\right)$, as for this term all atoms are already in the ground state. Furthermore, since only the relative geometrical phases play a role for the dynamics of the system, we can place the first atom at the coordinate origin, i.e., $\delta_{1, m}=0$ for $m \in\{1,2,3\}$ (see Fig. 1). Claiming $c_{G e} \in \mathbb{R}$ and denoting the complex phase of $c_{S g}$ and $c_{A g}$ as $\varphi_{S g / A g}=\operatorname{Arg}\left(\frac{c_{S g / A g}}{c_{G e}}\right)$, we find for the probability to detect at $\mathbf{r}_{3}$ the last photon spontaneously emitted by the three-atom ensemble being in the state $\hat{\rho}_{\text {sub }}$

$$
\begin{aligned}
G_{\hat{\rho}_{\text {sub }}}^{(1)}\left(t_{3}, \mathbf{r}_{3}\right) \propto & \left|c_{G e}\right|^{2} \mathrm{e}^{-2 \gamma t_{3}}+2\left|c_{S g}\right|^{2} \mathrm{e}^{-2(\gamma+\Delta \gamma) t_{3}} \cos ^{2}\left(\delta_{2,3} / 2\right)+2\left|c_{A g}\right|^{2} \mathrm{e}^{-2(\gamma-\Delta \gamma) t_{3}} \sin ^{2}\left(\delta_{2,3} / 2\right) \\
& +2\left|c_{S g}\right|\left|c_{A g}\right| \mathrm{e}^{-2 \gamma t_{3}} \sin \left(\delta_{2,3}\right) \sin \left(\varphi_{A g}-\varphi_{S g}+2 \Delta \Omega t_{3}\right) \\
& +2 \sqrt{2}\left|c_{S g}\right|\left|c_{G e}\right| \mathrm{e}^{-(2 \gamma+\Delta \gamma) t_{3}} \cos \left(\delta_{2,3} / 2\right) \cos \left(\varphi_{S g}+\delta_{2,3} / 2-\delta_{3,3}-\Delta \Omega t_{3}\right) \\
& +2 \sqrt{2}\left|c_{A g}\right|\left|c_{G e}\right| \mathrm{e}^{-(2 \gamma-\Delta \gamma) t_{3}} \sin \left(\delta_{2,3} / 2\right) \sin \left(\varphi_{A g}+\delta_{2,3} / 2-\delta_{3,3}+\Delta \Omega t_{3}\right) .
\end{aligned}
$$

Comparing to Eq. (5), we see from Eq. (9) that the addition of a third atom at a large distance from the first two atoms fundamentally changes the spatio-temporal behavior of $G_{\hat{\rho}_{\text {sub }}}^{(1)}\left(t_{3}, \mathbf{r}_{3}\right)$. In particular, the position of the third atom strongly affects the temporal behavior of the last three terms of Eq. (9), leading to a much more complex and rich structure of $G_{\hat{\rho}_{\text {sub }}}^{(1)}\left(t_{3}, \mathbf{r}_{3}\right)$ as discussed in detail in Appendix C. This is in contrast to the two-atom case where, for a given interatomic distance $R_{12}$, the temporal features of $G_{2 \text { atoms }}^{(1)}\left(t_{2}, \mathbf{r}_{2}\right)$ are unaffected by the atomic geometry. The system of three atoms therefore allows to fine tune the dynamics of the spontaneous decay along certain directions involving both temporal and spatial interferences alike.
Considering the birth of a photon, we can, for example, investigate the configuration where only the symmetric state of the first two atoms contributes to $G_{\hat{\rho}_{\text {sub }}}^{(1)}\left(t_{3}, \mathbf{r}_{3}\right)$ (i.e., $\left|c_{A g}\right|=0$ ); to achieve this we have to choose $\delta_{2,1}=\delta_{3,1}=\delta_{2,2}=\delta_{3,2}=$ $\delta=2 m \pi$, with $m \in \mathbb{Z}$ [see Eq. (7)]. Under these conditions, we find from Eq. (9)

$$
\begin{aligned}
G_{\hat{\rho}_{\text {sub }}}^{(1)}\left(t_{3}, \mathbf{r}_{3}\right) & \\
\propto & 4 \mathrm{e}^{-2 \gamma t_{3}}+16 \mathrm{e}^{-2(\gamma+\Delta \gamma) t_{3}} \cos ^{2}\left(\delta_{2,3} / 2\right) \\
& +16 \mathrm{e}^{-(2 \gamma+\Delta \gamma) t_{3}} \cos \left(\delta_{2,3} / 2\right) \cos \left(\delta_{2,3} / 2-\delta_{3,3}-\Delta \Omega t_{3}\right) .
\end{aligned}
$$


Equation (10) shows that, similar to the two-atom system, it is possible to find configurations where $G_{\hat{\rho}_{\text {sub }}}^{(1)}\left(t_{3}, \mathbf{r}_{3}\right)$ vanishes at $t_{3}=0$, e.g., by varying the position $\mathbf{r}_{3}$ of the third detector and/or the positions $\mathbf{R}_{2}$ and $\mathbf{R}_{3}$ of the second or third atom. (A similar expression as Eq. (10) is found if only the antisymmetric state of the first two atoms contributes to $G_{\hat{\rho}_{\text {sub }}}^{(1)}\left(t_{3}, \mathbf{r}_{3}\right)$, i.e., $\left|c_{S g}\right|=0$, see Appendix C.)

However, surprisingly and in difference to Eq. (5), beyond the birth of a photon, we can also obtain a death and a consecutive revival of a photon, by finding roots of $G_{\hat{\rho}_{\text {sub }}}^{(1)}\left(t_{3}, \mathbf{r}_{3}\right)$ for $t_{3}>0$.

The latter can be attained by setting $G_{\hat{\rho}_{\text {sub }}}^{(1)}\left(t_{3}, \mathbf{r}_{3}\right)=0$ in Eq. (10), in which case we get

$$
t_{S g}^{ \pm}=\frac{\ln \left( \pm 2 \cos \left(\delta_{2,3} / 2\right)\right)}{\Delta \gamma} .
$$

Note that in Eq. (11) it is required that $t_{S g}^{ \pm} \geqslant 0$ is real (what depends on the sign of $\Delta \gamma$ and the value of $\delta_{2,3}$ ). It then follows that

$$
\delta_{3,3}=\frac{\delta_{2,3}}{2}-\Delta \Omega t_{S g}^{ \pm}+\frac{\pi}{2}[(1+4 m) \pm 1] .
$$

Considering Eqs. (10)-(12), a complete disappearance of $G_{\hat{\rho}_{\text {sub }}}^{(1)}\left(t_{3}, \mathbf{r}_{3}\right)$ can be observed at times $t_{3}=t_{S g}>0$, followed by a reappearance and increase of $G_{\hat{\rho}_{\text {sub }}}^{(1)}\left(t_{3}, \mathbf{r}_{3}\right)$ for times $t_{3}>t_{S g}$ (see Fig. 2)

Our results may appear counter-intuitive, given that the death and revival of spontaneous emission are genuine features of a three-atom system and do not occur for two atoms. These effects, furthermore, only appear if the third atom is placed at a distance from the two nearby atoms, such that it does not interact with them. However, there is a simple intuitive explanation: the light emitted by two distant (noninteracting) atoms exhibits a spatial pattern, originating from a double-slit like interference, although for two distant atoms (i.e., without atom-atom interaction) there is no modified dynamics as compared to a single atom. In contrast, the temporal behavior of two (interacting) nearby atoms is altered via the dipole-dipole interaction, although there is no spatial dependency because the two atoms are close to each other. Our setup of two adjacent atoms together with a third distant atom uniquely combines the spatial and temporal interference effects in a minimal way, and it is this combination which leads to the qualitatively new phenomena we report.

In summary, we considered a setup in which the archetype system of two dipole-dipole interacting two-level atoms is augmented by a third identical atom, placed at a large distance compared to the transition wavelength $\lambda$ to the other two. Starting from initially excited atoms in free space, we have shown that the measurement of the first two spontaneously emitted photons may project the atomic ensemble into a singly-excited entangled state, which exhibits qualitatively different spontaneous emission features. In particular, the last emitted photon can exhibit a birth, i.e., a signal starting from zero at $t=0$ and increasing for $t>0$ up to a maximal value before dropping off exponentially thereafter. Moreover, the probability to detect the spontaneous photon can display a death followed by a revival for times $t>0$. The latter outcome is a genuine three-atom result, which does not appear in a two-atom system. These effects occur, since our three-atom system uniquely combines the spatial interference effects
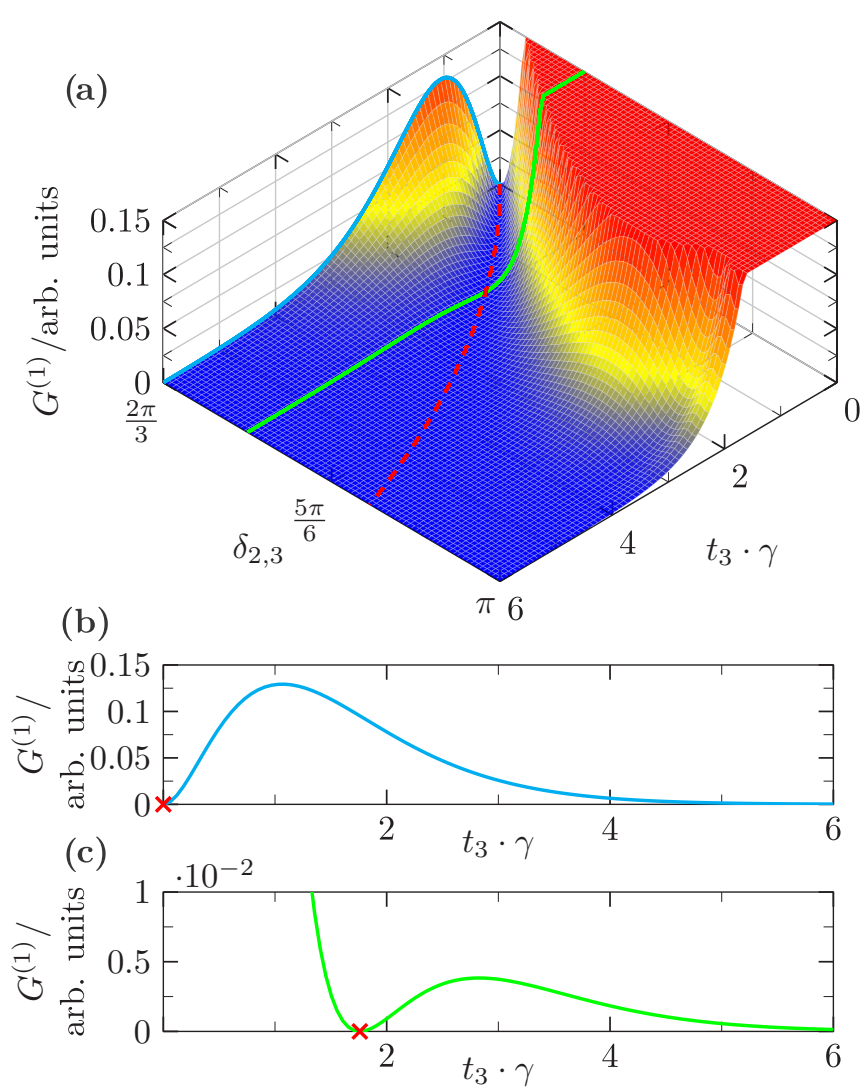

FIG. 2. (a) Probability $G_{\hat{\rho}_{\text {sub }}}^{(1)}\left(t_{3}, \mathbf{r}_{3}\right)$ of detecting the last photon spontaneously emitted by the three-atom system as a function of time $t_{3}$ and detector directions $\delta_{2,3}$ for the special case of the first two atoms being in a symmetric state. The roots $G_{\hat{\rho}_{\text {sub }}}^{(1)}\left(t_{3}, \mathbf{r}_{3}\right)=0$ are indicated by the red dashed line. The graph is limited to $G_{\hat{\rho}_{\text {sub }}}^{(1)}=0.15$ to visualize the region of of interest more clearly. [(b) and (c)] Probability $G_{\hat{\rho}_{\text {sub }}}^{(1)}\left(t_{3}, \mathbf{r}_{3}\right)$ in particular directions marked by the blue and green lines in (a), respectively. The red crosses mark times of zero detection probability, corresponding to a birth (b) and a death and revival (c), respectively. Parameters are $k_{0} R_{12}=\pi, \delta_{2,1}=\delta_{3,1}=$ $\delta_{2,2}=\delta_{3,2}=2 m \pi, \delta_{3,3}$ is given by Eq. (12), $\delta_{2,3}=2 \pi / 3$ for (b) and $\delta_{2,3}=3 \pi / 4$ for $(\mathrm{c})$.

observed in the emission from distant atoms with the temporal interference effects characteristic of nearby atoms.

Seen from a different perspective, $G_{\hat{\rho}_{\text {sub }}}^{(1)}\left(t_{3}, \mathbf{r}_{3}\right)$ corresponds to the third-order photon correlation function $G_{\hat{\rho}_{\text {exc }}}^{(3)}\left(0,0, t_{3} ; \mathbf{r}_{1}, \mathbf{r}_{2}, \mathbf{r}_{3}\right)$ of the fully excited atomic system [17]. Thus the death of a spontaneously emitted photon can also be viewed as a noncoincident three-photon Hong-Ou-Mandel interference, corresponding to the roots of $G_{\hat{\rho}_{\text {exc }}}^{(3)}\left(0,0, t_{3} ; \mathbf{r}_{1}, \mathbf{r}_{2}, \mathbf{r}_{3}\right)$ with appropriately positioned detectors. Here, similar to the original Hong-Ou-Mandel effect [24-30], the probability to measure multiple photons at different positions vanishes, yet with the difference that the detection occurs at different times $t_{1}=t_{2}=0$ and $t_{3}>0$.

The genuine three-atom phenomena reported here are not only of interest from a fundamental point of view. Since the third atom is far apart and thereby individually addressable, it provides a powerful tool to control the collective spontaneous emission of the total system at a distance. This could have 

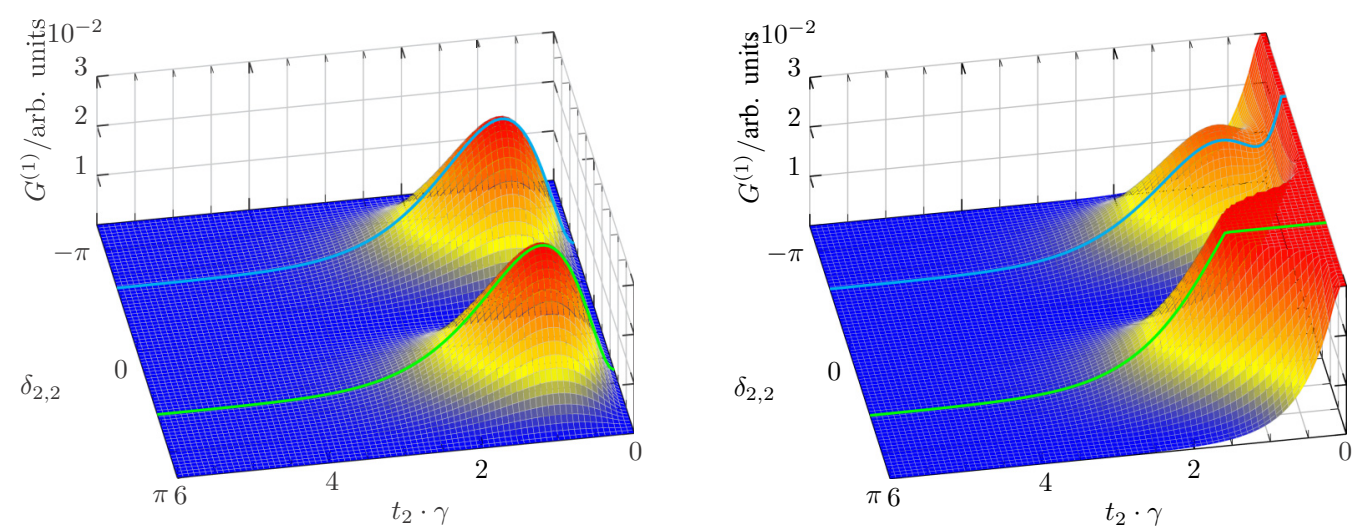

FIG. 3. Plot of the detection probability $G_{2 \text { atoms }}^{(1)}\left(t_{2}, \mathbf{r}_{2}\right)$ of the second photon as a function of time $t_{2}$ and detector position $\delta_{2,3}$ for $k_{0} R_{12}=\pi$. (Left) The condition $\delta_{2,1}=\delta_{2,2}+\pi(2 m+1)$ is fulfilled. The birth of $G_{2 \text { atoms }}^{(1)}\left(t_{2}, \mathbf{r}_{2}\right)$ for $t_{2}>0$ is clearly visible (blue and green lines). (Right) The condition is changed to $\delta_{2,1}=\delta_{2,2}+\pi(2 m+1)+\pi / 8$ leading to a superposition of birth (blue line) and the ordinary exponential decay (green line) in $G_{2 \text { atoms }}^{(1)}$ depending on the residual phase factor $\delta_{2,2}$.

a plethora of applications in fundamental science as well as in applied quantum technologies: the simple access and manipulation of the third atom-either via modification of its position or via illumination by an additional laser-could be used in quantum imaging, to extract spatial information with increased resolution of the two adjacent atoms which cannot be resolved easily due to their proximity. In turn, a laser-control of the relative phase of the distant atom can be used to control the directional emission.

\section{APPENDIX A: TIME EVOLUTION OF THREE ATOMS WITH TWO DIPOLE-DIPOLE-INTERACTING ATOMS}

The level splitting $\Delta \Omega$ and the rate $\Delta \gamma$ modifying the spontaneous emission rate from $2 \gamma$ to $2(\gamma \pm \Delta \gamma)$ of two dipole-dipole interacting atoms can be compactly written in the form [19-21]

$$
\begin{aligned}
\Delta \Omega+\mathrm{i} \Delta \gamma= & \frac{3}{2} \gamma \mathrm{e}^{-\mathrm{i} k_{0} R_{12}}\left[\frac{\cos ^{2}(\alpha)-1}{k_{0} R_{12}}+\left(1-3 \cos ^{2}(\alpha)\right)\right. \\
& \left.\times\left(\frac{\mathrm{i}}{\left(k_{0} R_{12}\right)^{2}}+\frac{1}{\left(k_{0} R_{12}\right)^{3}}\right)\right]
\end{aligned}
$$

where the angle $\alpha$ between the atomic dipole moment and $\mathbf{R}_{12}$ corresponds to $\pi / 2$ in our setup. As can be directly seen in Eq. (A1) both coupling constants depend on the interatomic distance $R_{12}$ and vanish for great atomic distances. Moreover, the master equation providing the time evolution of the threeatom system reads [19]

$$
\begin{aligned}
\partial_{t} \hat{\rho}= & -\mathrm{i} \omega_{0} \sum_{m=1}^{3}\left[\hat{\sigma}_{m}^{z}, \hat{\rho}\right]-\mathrm{i} \sum_{\substack{m, n=1 \\
m \neq n}}^{2} \Delta \Omega\left[\hat{\sigma}_{m}^{+} \hat{\sigma}_{n}^{-}, \hat{\rho}\right] \\
& -\sum_{m=1}^{3} \gamma\left(\hat{\sigma}_{m}^{+} \hat{\sigma}_{m}^{-} \hat{\rho}-2 \hat{\sigma}_{m}^{-} \hat{\rho} \hat{\sigma}_{m}^{+}+\hat{\rho} \hat{\sigma}_{m}^{+} \hat{\sigma}_{m}^{-}\right) \\
& -\sum_{\substack{m, n=1 \\
m \neq n}}^{2} \Delta \gamma\left(\hat{\sigma}_{m}^{+} \hat{\sigma}_{n}^{-} \hat{\rho}-2 \hat{\sigma}_{n}^{-} \hat{\rho} \hat{\sigma}_{m}^{+}+\hat{\rho} \hat{\sigma}_{m}^{+} \hat{\sigma}_{n}^{-}\right) .
\end{aligned}
$$

Note that by limiting the upper summation in the second and fourth terms in Eq. (A2) to 2, i.e., restricting the dipole-dipole interaction to the first two atoms, we treat the remote third atom as noninteracting.

\section{APPENDIX B: BIRTH OF SPONTANEOUS EMISSION IN A TWO-ATOM SYSTEM}

We show that a a system consisting of two atoms $G_{2 \text { atoms }}^{(1)}$ only shows a birth of spontaneous emission but does not display features such as death or revival as encountered for a three-atom system.

This means we have to show that $G_{2 \mathrm{atoms}}^{(1)}\left(t_{2}, \mathbf{r}_{2}\right)$, given in Eq. (5) of the main text and reprinted here

$$
\begin{aligned}
G_{2 \mathrm{atoms}}^{(1)}\left(t_{2}, \mathbf{r}_{2}\right) \propto & \mathrm{e}^{-2 \gamma t_{2}}\left(\mathrm{e}^{-2 \Delta \gamma t_{2}} \cos ^{2}\left(\delta_{2,1} / 2\right) \cos ^{2}\left(\delta_{2,2} / 2\right)\right. \\
& +\mathrm{e}^{2 \Delta \gamma t_{2}} \sin ^{2}\left(\delta_{2,1} / 2\right) \sin ^{2}\left(\delta_{2,2} / 2\right) \\
& \left.\left.+\sin \left(\delta_{2,1}\right) \sin \left(\delta_{2,2}\right) \frac{\cos \left(2 \Delta \Omega t_{2}\right)}{2}\right), \quad \text { B } 1\right)
\end{aligned}
$$

only vanishes if $t_{2}=0$, and that for $t_{2}>0$ we have $G_{2 \text { atoms }}^{(1)}\left(t_{2}, \mathbf{r}_{2}\right) \neq 0$.

We first focus on all time-dependent terms in Eq. (B1). Depending on the sign of $\Delta \gamma$, one of the exponentials in Eq. (B1), either $e^{\Delta \gamma t}$ or $e^{-\Delta \gamma t}$, increases for $t_{2}>0$ and becomes greater than one, whereas the last term in Eq. (B1) oscillates as a function of $t_{2}$, with a maximum amplitude equal to one. Since $\Delta \gamma$ and $\Delta \Omega$ are of the same order of magnitude (for a given atomic separation $R_{12}$ ), we only have to focus on the interval $0<t_{2}<\Delta \Omega \pi / 2$. In fact, only for $t_{2}$ close to zero it is possible to achieve $G_{2 \text { atoms }}^{(1)}\left(t_{2}, \mathbf{r}_{2}\right)=0$ if and only if $\sin \left(\delta_{2,1}\right) \sin \left(\delta_{2,2}\right)$ is negative. For $t_{2}=0$ the oscillating term in Eq. (B1) is maximal and Eq. (B1) reduces to

$$
\begin{aligned}
& G_{2 \text { atoms }}^{(1)}\left(t_{2}, \mathbf{r}_{2}\right) \\
& \quad \propto \cos ^{2}\left(\delta_{2,1} / 2\right) \cos ^{2}\left(\delta_{2,2} / 2\right) \\
& \quad+\sin ^{2}\left(\delta_{2,1} / 2\right) \sin ^{2}\left(\delta_{2,2} / 2\right)+\frac{1}{2} \sin \left(\delta_{2,1}\right) \sin \left(\delta_{2,2}\right) .
\end{aligned}
$$


Now, claiming $G_{2 \text { atoms }}^{(1)}=0$ in Eq. (B2), we find the condition $\delta_{2,1}=\delta_{2,2}+\pi(2 m+1), m \in \mathbb{Z}$ and with that we obtain $\sin \left(\delta_{2,1}\right) \sin \left(\delta_{2,2}\right)=-\sin \left(\delta_{2,2}\right)^{2}$. For $t_{2}>0$, we then have

$$
\begin{aligned}
& G_{2 \text { atoms }}^{(1)}\left(t_{2}, \mathbf{r}_{2}\right) \\
& \quad \propto e^{-2 \gamma t} \sin ^{2}\left(\delta_{2,2}\right)(\cosh (2 \Delta \gamma t)-\cos (2 \Delta \Omega t)) .
\end{aligned}
$$

Equation (B3) shows that $t_{2}=0$ is indeed the only solution for $G_{2 \text { atoms }}^{(1)}=0$, i.e., it exists no point in time $t_{2}^{*}>0$ for which $G_{2 \text { atoms }}^{(1)}\left(t_{2}^{*}, \mathbf{r}_{2}\right)=0$, except the trivial solution $\delta_{2,1}=\delta_{2,2}+$ $\pi(2 m+1)$ and $\delta_{2,2}=n \pi, n \in \mathbb{Z}$ for which $G_{2 \text { atoms }}^{(1)}\left(t_{2}, \mathbf{r}_{2}\right)=0$ for all $t_{2}$.

Again we want to point out, that it is sufficient to restrict the analysis to the case $\delta_{2,1}=\delta_{2,2}+\pi(2 m+1)$ because for all other choices of $\delta_{1,2}$ and $\delta_{2,2}$ the first two terms in Eq. (B1) are already greater than the third term for $t_{2}=0$ and thus for all $t_{2}>0$.

As can be seen in Fig. 3, for an arbitrary choice of $\delta_{2,1}$ and $\delta_{2,2}, G_{2 \text { atoms }}^{(1)}$ in general shows both, a birth of spontaneous emission, an exponential decay, or a superposition of both features.

\section{APPENDIX C: DEATH AND REVIVAL IN A THREE-ATOM SYSTEM}

In the main text, we derived in Eq. (9) the detection probability of the last spontaneously emitted photon depending on the actual two-photon reduced atomic state $\hat{\rho}_{\text {sub }}$. We then investigated the special case for the first two atoms being in a symmetric state which results in Eq. (10). The so called death and subsequent revival of spontaneous emission can be found along the detection directions at a given detection time given by Eqs. (11) and (12), respectively. Similarly we can set $\left|c_{S g}\right|=0$ and examine the antisymmetric state of the first two atoms. To achieve this configuration, we have to set $\delta_{2,1}=\delta_{3,1}=\delta_{2,2}=\delta_{3,2}=\pi(2 m+1)$ with $m \in \mathbb{Z}$ and thus obtain

$$
\begin{aligned}
& G_{\hat{\rho}_{\text {sub }}}^{(1)}\left(t_{3}, \mathbf{r}_{3}\right) \\
& \propto 4 \mathrm{e}^{-2 \gamma t_{3}}+16 \mathrm{e}^{-2(\gamma-\Delta \gamma) t_{3}} \sin ^{2}\left(\delta_{2,3} / 2\right) \\
& \quad+16 \mathrm{e}^{-(2 \gamma-\Delta \gamma) t_{3}} \sin \left(\delta_{2,3} / 2\right) \sin \left(\delta_{2,3} / 2-\delta_{3,3}+\Delta \Omega t_{3}\right) .
\end{aligned}
$$

Comparing Eq. (C1) with Eq. (10) of the paper both expression are identical after performing the transitions $\Delta \gamma \rightarrow$ $-\Delta \gamma, \Delta \Omega \rightarrow-\Delta \Omega$, and $\sin \rightarrow \cos$. Thus we find true roots of Eq. (C1) for times

$$
t_{A g}^{ \pm}=\frac{\ln \left( \pm 2 \sin \left(\delta_{2,3} / 2\right)\right)}{\Delta \gamma}
$$

where we have to choose the sign such that $t_{A g}>0$ is real. For the geometrical phase $\delta_{3,3}$, it then follows that

$$
\delta_{3,3}=\frac{\delta_{2,3}}{2}-\Delta \Omega t_{A g}^{ \pm}+\frac{\pi}{2}[4 m \pm 1]
$$

Although the three-atom system also produces a birth of spontaneous emission (Fig. 4) similar to the two-atom case,
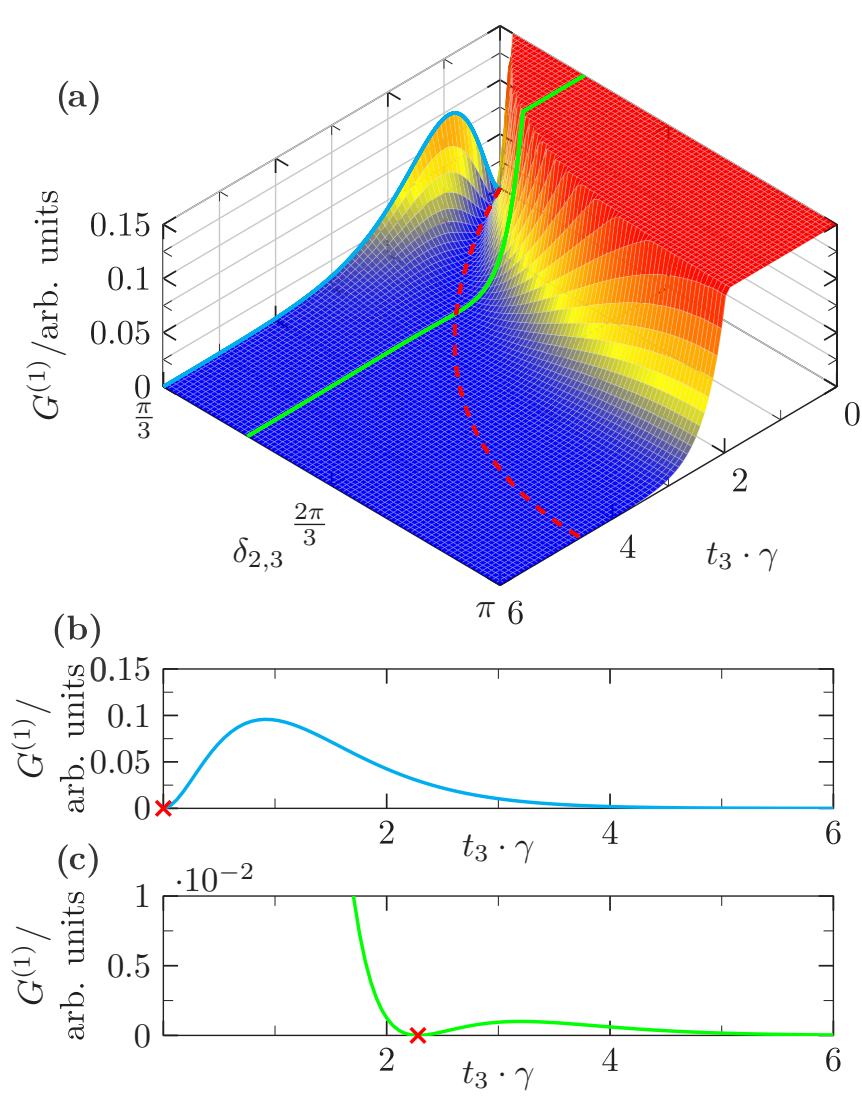

FIG. 4. (a) Probability $G_{\hat{\rho}_{\text {sub }}}^{(1)}\left(t_{3}, \mathbf{r}_{3}\right)$ of detecting the last photon spontaneously emitted by the three-atom system as a function of time $t_{3}$ and detector directions $\delta_{2,3}$ for the special case of the first two atoms being in an antisymmetric state. The roots $G_{\hat{\rho}_{\text {sub }}}^{(1)}\left(t_{3}, \mathbf{r}_{3}\right)=0$ are indicated by the red dashed line. The graph is limited to $G_{\hat{\rho}_{\text {sub }}}^{(1)}=0.15$ to visualize the region of of interest more clearly. [(b) and (c)] Probability $G_{\hat{\rho}_{\text {sub }}}^{(1)}\left(t_{3}, \mathbf{r}_{3}\right)$ in particular directions marked by the blue and green lines in (a), respectively. The red crosses mark times of zero detection probability, corresponding to a birth (b) and a death and revival (c). Parameters are $k_{0} R_{12}=\pi, \delta_{2,1}=\delta_{3,1}=\delta_{2,2}=$ $\delta_{3,2}=(2 m+1) \pi, \delta_{3,3}$ is given by Eq. (C3), $\delta_{2,3}=\pi / 3$ for (b) and $\delta_{2,3}=\pi / 2$ for (c).

$G_{\hat{\rho}_{\text {sub }}}^{(1)}$ in Eq. (C1) differs significantly from Eq. (B1). In the latter equation, the third interference term only depends on $\cos (\Delta \Omega t)$ whereas in Eq. (C1) we find $\sin \left(\delta_{2,3} / 2-\delta_{3,3}+\right.$ $\left.\Delta \Omega t_{3}\right)$ which opens up the possibility to highlight all temporal features via tuning the geometrical phases $\delta_{2,3}$ and $\delta_{3,3}$. As a consequence, for $\delta_{2,3} / 2-\delta_{3,3}<0$, the major contribution of the interference term in Eq. (C1) is shifted towards grater times $t_{3}$, i.e., the death of spontaneous emission is shifted from $t_{3}=0$ to $t_{3}>0$ followed by a revival directly thereafter.

Finally, we want to give a physical interpretation for all terms occurring in Eq. (11) of the paper. (1) The first three terms correspond to the photon detection probability produced by each individual state $|G, e\rangle,|S, g\rangle$, and $|A, g\rangle$. (2) The fourth term corresponds to the interference between the symmetric 
and antisymmetric state. It oscillates with $2 \Delta \Omega$ due to the level splitting. (3) The fifth and sixth terms correspond to the interference between the symmetric and antisymmetric state with the independent atom, respectively. It oscillates with frequency $\pm \Delta \Omega$ due to the level splitting.
By setting either $\left|c_{S g}\right|=0$ or $\left|c_{A g}\right|=0$, Eq. (11) is reduced by three terms in each case. Thus investigating the more complex case for which both $\left|c_{S g}\right| \neq 0$ and $\left|c_{A g}\right| \neq 0$ may give rise to a multitude of additional fascinating and interesting regimes.
[1] E. T. Jaynes and F. W. Cummings, Comparison of quantum and semiclassical radiation theories with application to the beam maser, Proc. IEEE 51, 89 (1963).

[2] J. H. Eberly, N. B. Narozhny, and J. J. Sanchez-Mondragon, Physical Review, Phys. Rev. Lett. 44, 1323 (1980).

[3] N. B. Narozhny, J. J. Sanchez-Mondragon, and J. H. Eberly, Coherence versus incoherence: Collapse and revival in a simple quantum model, Phys. Rev. A 23, 236 (1981).

[4] G. Rempe, H. Walther, and N. Klein, Observation of Quantum Collapse and Revival in a One-Atom Maser, Phys. Rev. Lett. 58, 353 (1987).

[5] M. Brune, F. Schmidt-Kaler, A. Maali, J. Dreyer, E. Hagley, J. M. Raimond, and S. Haroche, Quantum Rabi Oscillation : A Direct Test of Field Quantization in a Cavity, Phys. Rev. Lett. 76, 1800 (1996).

[6] E. L. Hahn, Spin echoes, Phys. Rev. 80, 580 (1950).

[7] M. Greiner, O. Mandel, T. W. Hänsch, and I. Bloch, Collapse and revival of the matter wave field of a bose-einstein condensate, Nature (London) 419, 51 (2002).

[8] R. R. Puri and G. S. Agarwal, Collapse and revival phenomena in the jaynes-cummings model with cavity damping, Phys. Rev. A 33, 3610 (1986).

[9] T. Yu and J. H. Eberly, Sudden death of entanglement, Science 323, 598 (2009).

[10] T. Yu and J. H. Eberly, Finite-Time Disentanglement Via Spontaneous Emission, Phys. Rev. Lett. 93, 140404 (2004).

[11] Z. Ficek and R. Tanaś, Dark periods and revivals of entanglement in a two-qubit system, Phys. Rev. A 74, 024304 (2006).

[12] M. Yönaç, T. Yu, and J. H. Eberly, Sudden death of entanglement of two Jaynes Cummings atoms, J. Phys. B 39, S621 (2006).

[13] I. Sainz and G. Björk, Entanglement invariant for the double Jaynes-Cummings model, Phys. Rev. A 76, 042313 (2007).

[14] C. Thiel, J. von Zanthier, T. Bastin, E. Solano, and G. S. Agarwal, Generation of Symmetric Dicke States of Remote Qubits with Linear Optics, Phys. Rev. Lett. 99, 193602 (2007).

[15] T. Bastin, C. Thiel, J. von Zanthier, L. Lamata, E. Solano, and G. S. Agarwal, Operational Determination of Multiqubit Entanglement Classes Via Tuning of Local Operations, Phys. Rev. Lett. 102, 053601 (2009).

[16] S. Oppel, R. Wiegner, G. S. Agarwal, and J. von Zanthier, Directional Superradiant Emission from Statistically Independent Incoherent Nonclassical and Classical Sources, Phys. Rev. Lett. 113, 263606 (2014).

[17] R. Wiegner, S. Oppel, D. Bhatti, J. von Zanthier, and G. S. Agarwal, Simulating superradiance from higher-order- intensity-correlation measurements: Single atoms, Phys. Rev. A 92, 033832 (2015).

[18] W. Feng, Y. Li, and S.-Y. Zhu, Cooperative spontaneous emission of three identical atoms, Phys. Rev. A 88, 033856 (2013).

[19] G. S. Agarwal, Quantum Statistical Theories of Spontaneous Emission and Their Relation to Other Approaches, edited by G. Möhler et al., Springer Tracts in Modern Physics: Quantum Optics Vol. 70 (Springer-Verlag, Berlin, 1974).

[20] W. T. Rhodes, T. Asakura, K.-H. Brenner, T. W. Hänsch, T. Kamiya, F. Krausz, B. Monemar, H. Venghaus, H. Weber, and $\mathrm{H}$. Weinfurter, eds., Quantum Interference and Coherence (Springer-Verlag, Berlin, 2005).

[21] M. Kiffner, M. Macovei, J. Evers, and C. H. Keitel, Vacuuminduced processes in multilevel atoms, Prog. Opt. 55, 85 (2010).

[22] Z. Ficek, R. Tanaś, and S. Kielich, Quantum beats in photon correlations of spontaneous emission from two nonidentical atoms, Sov. J. Quantum Electron. 18, 766 (1988).

[23] J. von Zanthier, T. Bastin, and G. S. Agarwal, Measurementinduced spatial modulation of spontaneous decay and photon arrival times, Phys. Rev. A 74, 061802(R) (2006).

[24] C. K. Hong, Z. Y. Ou, and L. Mandel, Measurement of Subpicosecond Time Intervals between Two Photons by Interference, Phys. Rev. Lett. 59, 2044 (1987).

[25] Y. H. Shih and C. O. Alley, New Type of Einstein-PodolskyRosen-Bohm Experiment using Pairs of Light Quanta Produced by Optical Parametric Down Conversion, Phys. Rev. Lett. 61, 2921 (1988).

[26] J. Beugnon, M. P. A. Jones, J. Dingjan, B. Darquié, G. Messin, A. Browaeys, and P. Grangier, Quantum interference between two single photons emitted by independently trapped atoms, Nature (London) 440, 779 (2006).

[27] J. Gillet, G. S. Agarwal, and T. Bastin, Tunable entanglement, antibunching, and saturation effects in dipole blockade, Phys. Rev. A 81, 013837 (2010).

[28] R. Wiegner, C. Thiel, J. von Zanthier, and G. S. Agarwal, Creating path entanglement and violating bell inequalities by independent photon sources, Phys. Lett. A 374, 3405 (2010).

[29] R. B. Patel, A. J. Bennett, I. Farrer, C. A. Nicoll, D. A. Ritchie, and A. J. Shields, Two-photon interference of the emission from electrically tunable remote quantum dots, Nature Photonics 4, 632 (2010).

[30] J. Hofmann, M. Krug, N. Ortegel, L. Gérard, M. Weber, W. Rosenfeld, and H. Weinfurter, Heralded entanglement between widely separated atoms, Science 337, 72 (2012). 